The ASTrophysical Journal SUPPLEMENT SERIES, 90:863-868, 1994 February

(C) 1994. The American Astronomical Society. All rights reserved. Printed in U.S.A.

\title{
GAMMA-RAY BURST THEORY: BACK TO THE DRAWING BOARD
}

\author{
ALICE K. HARDING \\ Laboratory for High Energy Astrophysics, NASA/Goddard Space Flight Center, Greenbelt, MD 20771 \\ Received 1993 March 8; accepted 1993 May 19
}

\begin{abstract}
Gamma-ray bursts have always been intriguing sources to study in terms of particle acceleration, but not since their discovery two decades ago has the theory of these objects been in such turmoil. Prior to the launch of Compton Gamma-Ray Observatory and observations by BATSE, there was strong evidence pointing to magnetized Galactic neutron stars as the sources of gamma-ray bursts. However, since BATSE the observational picture has changed dramatically, requiring much more distant and possibly cosmological sources. I review the history of gamma-ray burst theory from the era of growing consensus for nearby neutron stars to the recent explosion of halo and cosmological models and the impact of the present confusion on the particle acceleration problem.
\end{abstract}

Subject headings: acceleration of particles - gamma rays: bursts

\section{INTRODUCTION}

The origin of gamma-ray bursts (GRBs) is one of the most challenging puzzles in astrophysics and one which has gone unsolved for two decades. Our lack of understanding of these sources persists because no convincing counterparts have been discovered at other wavelengths and because their isotropic spatial distribution does not reveal any distance scale. In the 5 years prior to the launch of the Compton Gamma-Ray Observatory, hopes were raised briefly that we were getting closer to understanding the sources of bursts. Accumulating evidence for Galactic neutron stars as the sources seemed to culminate in the detection by Ginga of double harmonic absorption-line features in three GRB spectra (Murakami et al. 1988), confirming that cyclotron scattering was occurring in magnetic fields near $10^{12} \mathrm{G}$. It was thought that if GRBs were indeed a Galactic disk population of neutron stars then the BATSE detector on $C G R O$, with significantly increased sensitivity to weak bursts would begin to see an anisotropy in the spatial distribution toward the Galactic plane. To almost everyone's surprise, BATSE has seen an extremely isotropic source distribution, even for the faintest bursts (Meegan et al. 1991). At the same time, the count spectrum flattens at the low end, indicating a decrease in source density at low fluence, or an edge to the distribution. At least for most of the bursts, models for nearby Galactic neutron stars now appear to be ruled out, and the mystery has deepened. Models which are compatible with the BATSE distribution involve sources at distances of at least 50 $\mathrm{kpc}$ and burst energies in $\gamma$-rays of at least $10^{42}$ ergs $^{-1}$.

I will review the present status of gamma-ray burst theory, with emphasis on the general problems shared by all models, that is, generation of a large amount of energy in a small volume and production of a hard, nonthermal spectrum. I will also discuss how some models are attempting to overcome these problems and predictions of the models which may ultimately be tested by further observations.

\section{PRE-BATSE GAMMA-RAY BURST THEORY}

Observations of GRBs over the two decades prior to $C G R O$ built up a considerable body of circumstantial evidence point- ing to magnetized neutron stars as the origin of the bursts. (1) Intensity fluctuations on submillisecond time scales in burst time histories indicate a source size less than tens of kilometers. (2) The presence of line features at energies between 20 and $60 \mathrm{keV}$ (Mazets et al. 1981; Heuter 1988; Murakami et al. 1988) are explained quite consistently by cyclotron scattering in strong neutron star magnetic fields (Wang et al. 1989; Harding \& Preece 1989). In addition, less significant evidence for emission features around $400-450 \mathrm{keV}$ in some GRB spectra could be electron-positron pair annihilation lines at 511 $\mathrm{keV}$, redshifted by a neutron star gravitational field. (3) Lack of quiescent counterparts at GRB locations put stringent limits on the nonbursting flux and are compatible with an isolated compact object. (4) Soft X-ray tails and precursors observed in a few GRBs can be fitted with blackbody spectra at temperatures of a few keV (Murakami et al. 1991) and give a source size estimate of $R \sim 0.7(d / 1 \mathrm{kpc}) \mathrm{km}$ which is consistent with a neutron star polar region.

Given these strong indications, a number of models were developed for GRBs originating on magnetic neutron stars in the Galaxy. Those which have been studied most seriously include the impact of a comet or other solid body onto a neutron star, a thermonuclear flash due to slow accretion, and a starquake or glitch ( see Harding 1991 for complete review and references ).

\section{POST-BATSE GAMMA-RAY BURST THEORY}

\subsection{Source Distribution}

BATSE has now detected over $600 \mathrm{GRBs}$ with localizations on the sky to as small as $2^{\circ}$ accuracy. The spatial distribution of these bursts has been found to be isotropic to a very high degree, with measured dipole moment of $\langle\cos \theta\rangle=0.034 \pm$ 0.027 and quadrupole moment $\langle\sin b\rangle=0.316 \pm 0.014$ (Meegan et al. 1993). The expected values for an isotropic distribution, including effects of nonuniform sky coverage, are $\langle\cos \theta\rangle=-0.014$ and $\langle\sin b\rangle=0.329$. (Note that there is presently a $1.8 \sigma$ deviation from isotropy in the dipole moment.) At the same time the count spectrum of these bursts, or 
the distribution of $\log N\left(>C_{\max } / C_{\min }\right)-\log C_{\max } / C_{\min }$ where $C_{\max }$ is the maximum count rate of the burst and $C_{\min }$ is the detection threshold, follows the $-3 / 2$ power law expected for a uniform source distribution at high $C_{\max } / C_{\min }$, but flattens to a -1 power law at low $C_{\max } / C_{\min }$. This indicates that the number of sources is decreasing at large distances or that we are seeing an edge to the source distribution. This rules out sources that are distributed in a disk with scale height of a few hundred parsecs, which would exhibit some anisotropy if we were seeing beyond the edge of the disk (Paczyński 1991a). The source distributions that are now compatible with the BATSE data include a local population such as the Oort cloud $\left(d \sim 10^{4}\right.$ $10^{5} \mathrm{AU}$ ), an extended Galactic halo population, or a population of sources at cosmological distances.

From a purely geometrical point of view, a cosmological distribution of GRB sources is the simplest distribution that is consistent with the BATSE data. Sources which uniformly fill the observable volume of the universe are perfectly isotropic and will exhibit a natural decrease in source density due to redshift effects at $z>1$. Simple cosmological source models without evolution can fit the BATSE count spectrum and imply an average source luminosity of $L \sim 4-5 \times 10^{51} \mathrm{ergs} \mathrm{s}^{-1}$ (Dermer 1992; Mao \& Paczyński 1992; Piran 1992). However, Fenimore et al. (1992) caution that including the source count data from PVO will significantly change the fitting parameters of cosmological models. This is because PVO has observed many more high-fluence GRBs during its 14 year lifespan and has therefore sampled several decades higher into the $-3 / 2$ region of the count spectrum than BATSE. Fits of cosmological source models to a combined BATSE/PVO count spectrum gives a lower average source luminosity of $\sim 6 \times 10^{50} \mathrm{ergs} \mathrm{s}^{-1}$ (Fenimore et al. 1993b).

A Galactic halo distribution of sources can also fit the count data and not violate the isotropy constraints. However, generic halo models with an assumed density distribution, $\rho \propto\left(R_{c}^{2}+\right.$ $\left.R^{2}\right)^{-1}$ where $R_{c}$ is the core radius, must have an extent of at least 50 kpc (Brainerd 1992a; Paczyński 1991a). The most recent analysis using the distribution of 400 BATSE bursts finds that halo models must have core radii larger than $34 \mathrm{kpc}$, sampled to at least $140 \mathrm{kpc}$ (Hakkila et al. 1993). This is larger than the dark matter halo of our Galaxy and does not correspond to any other known Galactic source distribution. It has been suggested though that we may already have seen the objects that are responsible for such extended halos of GRBs, namely high-velocity neutron stars (Eichler \& Silk 1992; Hartmann, Linder, \& The 1993; Li \& Dermer 1992). A number of radio pulsars have been observed with space velocities exceeding $300 \mathrm{~km} \mathrm{~s}^{-1}$ (Harrison, Lyne, \& Anderson 1993), including several with velocities exceeding escape velocity from the Galaxy $\left(\sim 600 \mathrm{~km} \mathrm{~s}^{-1}\right)$. These neutron stars could either be born in the disk and populate an extended halo during their lifetimes, or they could be a separate population of neutron stars born in the halo. Those that are born in the disk and are escaping from the Galaxy must have a delayed turn-on as GRB sources to avoid violating the BATSE isotropy constraint ( $\mathrm{Li} \&$ Dermer 1992). One problem with the hypothesis that highvelocity neutron stars are born in the disk is that several pulsars with high velocities have been observed moving toward the Galactic plane (Lyne, Anderson, \& Salter 1982).

\subsection{Relativistic Fireballs}

Both Galactic halo and cosmological models for GRB sources share a fairly serious problem that did not plague the disk models: very high optical depth to photon-photon pair production for isotropic radiation. As mentioned before, the short time scales observed for intensity fluctuations in GRBs implies a source size of $R<10^{7} \mathrm{~cm}$. This small size together with the large implied burst luminosities require high isotropic photon densities at the source. The optical depth to photonphoton pair production in the source can be estimated from the required photon density:

$$
\tau_{\gamma \gamma} \simeq n_{\gamma} \sigma_{\mathrm{T}} R=\left(\frac{L}{R}\right) \frac{\sigma_{\mathrm{T}}}{4 \pi m c^{3}}
$$

where $\sigma_{\mathrm{T}}$ is the Thomson cross section. The luminosity of a burst with observed flux $\phi$, emitting isotropically, is $L \simeq 10^{37}$ ergs $\mathrm{s}^{-1}(d / 100 \mathrm{pc})^{2}\left(\phi / 10^{-5}\right.$ ergs $\left.\mathrm{cm}^{-2} \mathrm{~s}^{-1}\right)$. For nearby sources in the disk at distance $d \sim 100 \mathrm{pc},\langle L\rangle \sim 10^{37} \mathrm{ergs} \mathrm{s}^{-1}$ and $\tau_{\gamma \gamma} \sim 1$. For sources in an extended Galactic halo at $d \sim$ $100 \mathrm{kpc},\langle L\rangle \sim 10^{43} \mathrm{ergs} \mathrm{s}^{-1}$ and $\tau_{\gamma \gamma} \sim 10^{6}$, while for sources at cosmological distances of $d \sim 100 \mathrm{Mpc},\langle L\rangle \sim 10^{49} \mathrm{ergs}$ $\mathrm{s}^{-1}$ and $\tau_{\gamma \gamma} \sim 10^{12}$. Pair production will produce a turnover or cutoff in the observed spectrum around $1 \mathrm{MeV}$, which is not seen in most GRB spectra, many of which extend to at least 10 $\mathrm{MeV}$. The lack of an observed cutoff in observed GRB spectra, implying that $\tau_{\gamma \gamma}<1$, was originally used to place a limit of a few kpc on the distance to the sources (Schmidt 1978). BATSE has observed spectral breaks between $400 \mathrm{keV}$ and 2 $\mathrm{MeV}$ in several GRBs (Schaefer et al. 1992) which could be due to pair production. However, this interpretation has problems for cosmological sources and probably even Galactic sources (Baring 1993; Baring \& Harding 1993), as will be discussed later.

If $\tau_{\gamma \gamma} \gg 1$, as would be the case if GRB sources at extended halo or cosmological distances are emitting isotropically, not only would the spectra be absorbed above $1 \mathrm{MeV}$ but the large numbers of pairs produced would make the source optically thick to Compton scattering as well. This would result in a thermalized pair plasma, emitting a blackbody spectrum at a temperature $k T \sim m c^{2}$, very different from the power-law spectra observed from GRBs. Goodman (1986) pointed out that an optically thick pure electron-positron pair plasma at this temperature would expand adiabatically as a relativistic fluid, cooling as $T \propto 1 / R$, where $R$ is the source size increasing as a function of time. The behavior of these so-called relativistic fireballs is thus analogous to the expansion of the universe. The fireball will expand and cool as thermal energy is converted to bulk kinetic energy until it becomes optically thin and the radiation can escape. At this point the internal temperature is $k T_{\text {esc }} \sim 20 \mathrm{keV}$ and the Lorentz factor of the expansion is $\Gamma \approx R_{\text {esc }} / R_{0}$ (Paczyński 1986). The escaping radiation is thus blueshifted in the observer's frame to $h v_{\text {obs }} \simeq \Gamma T_{\text {esc }}=T_{0}$, where $R_{0}$ and $T_{0}$ are the initial radius and temperature of the fireball. The asymptotic value of $\Gamma$ depends on the luminosity of the fireball. For a cosmic fireball, $\Gamma \sim 10^{2}-10^{4}$, while for a GRB fireball in the Galactic halo, $\Gamma \sim 10$ (Piran \& Shemi 1992). So, one avoids the photon-photon attenuation of the 
spectrum, but the escaping radiation is a (slightly) modified blackbody at a temperature of a few $\mathrm{MeV}$ in the cosmic fireball case.

A further problem that arises is that if the fireball contains even a small number of baryons, which would seem hard to avoid, their inertia can dominate and change the dynamics (Shemi \& Piran 1990). If the ratio of baryon rest mass to total energy of the fireball, $10^{-8}<M c^{2} / E_{0}<10^{-5}$, the baryons will dominate the opacity but will not affect the dynamics. However, if $M c^{2} / E_{0}>10^{-5}$, most of the energy of the fireball will go into accelerating the baryons and cannot be reconverted to radiation. The end result will be relativistically expanding baryons with $\Gamma \approx E_{0} / M c^{2}$ and a quenched GRB with very low radiation efficiency.

\subsection{Possible Solutions to the Fireball Problem}

There have been several suggested ways to avoid the GRB fireball problem. One solution would be to prevent a fireball in the first place, that is, the GRB must not go through a phase in which the source is optically thick to pair production. This can be accomplished in several ways. First, anisotropy of the radiation field would decrease $\tau_{\gamma \gamma}$ by increasing the pair production threshold, which depends on the angle a photon propagates with respect to other photons. The threshold for a photon of energy $\epsilon_{1}$ to produce a pair with a second photon of energy $\epsilon_{2}$ is

$$
\epsilon_{1}=2 m^{2} c^{4} /\left(1-\cos \theta_{12}\right) \epsilon_{2},
$$

where $\theta_{12}$ is the angle between the two photons. For smaller $\theta_{12}$, the target photons are at higher energies, where photon densities (e.g., in the case of a power-law spectrum) are lower. In the case of GRBs at either halo or cosmological distances, the inferred optical depth for an isotropic radiation field is so high that the radiation from the source must be highly beamed to avoid $\tau_{\gamma \gamma}>1$.

A second way to avoid high pair production optical depth is to have relativistic motion of the source. Since this will automatically produce beamed radiation, it is really a special case of radiation anisotropy, which in general does not require motion of the source. There will be a reduction in $\tau_{\gamma \gamma}$ due to both a reduction of the required photon density and photon energy in the comoving frame (Krolik \& Pier 1991):

$$
\tau_{\gamma \gamma}(\epsilon) \simeq 10^{2}(2 \Gamma)^{-(1+2 \alpha)} \epsilon^{\alpha-1} d_{\mathrm{kpc}}^{2}\left(\frac{\phi}{10^{-7} \operatorname{ergs~cm} \mathrm{cm}^{-2} \mathrm{~s}^{-1}}\right) \frac{\ell_{6}}{A_{12}}
$$

where $\Gamma$ is the bulk Lorentz factor, $\alpha$ is the photon spectral index, $\ell_{6} \equiv \ell / 10^{6} \mathrm{~cm}$ and $A_{12} \equiv A / 10^{12} \mathrm{~cm}^{2}$ are the thickness and cross-sectional area of the source, $d_{\mathrm{kpc}} \equiv d / 1 \mathrm{kpc}$ is the distance, and $\epsilon$ is the photon energy in units of $m c^{2}$. This reduction of $\tau_{\gamma \gamma}$ is equivalent to that derived for beamed radiation due to the increase in threshold, given that the beaming angle is inversely proportional to the bulk Lorentz factor, $\theta_{B} \sim$ $1 / \Gamma$. The bulk Lorentz factor required to make $\tau_{\gamma \gamma}<1$ at energy $\epsilon$ for a source with $\phi=10^{-7}$ ergs $\mathrm{cm}^{-2} \mathrm{~s}^{-1}$ and $\ell_{6}=$ $A_{12}=1$ is (Baring 1993)

$$
\Gamma^{1+2 \alpha} \gtrsim 39 d_{\mathrm{kpc}}^{2}[(1+z) \epsilon]^{\alpha-1} \frac{1}{\alpha^{5 / 3}(4 / 3+\alpha)^{27 / 11}}
$$

where a cosmological redshift $z$ of the observed photon energy has been included. So at $d=100 \mathrm{kpc}$, Lorentz factors $\Gamma \gtrsim 36$ and $\Gamma \gtrsim 6$ are required at $\epsilon=1$ for $\alpha=1$ and 2 , while at $d=100$ Mpc, $\Gamma \gtrsim 4 \times 10^{3}$ and $\Gamma \gtrsim 10^{2}$ are required for $\alpha=1$ and 2 . The Lorentz factors required to make cosmological GRBs optically thin are several orders of magnitude higher than Lorentz factors, $\Gamma \sim 5-10$, of jets associated with active galactic nuclei, as inferred from observations of superluminal expansion and BL Lac sources.

Four GRBs have been detected to energies well above 1 MeV by the EGRET detector on CGRO: GRB 910503 to 170 MeV (Schneid et al. 1992), GRB 910601 and GRB 910814 (Kwok et al. 1993) and GRB 930131 to $1 \mathrm{GeV}$ (Sommer et al. 1993). The bulk Lorentz factors from eqn (4) required to allow the escape of the high-energy photons from these sources are even higher and for three of the bursts, relativistic motion with $\Gamma \simeq 2.5-3$ is required even at $d=150$ pc (Baring \& Harding 1993). The maximum distances that are consistent with isotropic radiation are a few parsecs. Furthermore, the spectral breaks observed around $1 \mathrm{MeV}$ cannot be explained by pair production, because the high $\Gamma$ would blueshift such breaks to energies $\gg 1 \mathrm{MeV}$ (Baring 1993).

Although the above calculations have assumed that the radiation is beamed within angle $1 / \mathrm{r}$, such a narrow beam is not necessary to make the source optically thin. Fenimore, Epstein, \& Ho (1993a) have shown that a relativistically expanding, thin shell will allow escape of high-energy gamma rays and presumably a range of intermediate source geometries between a narrow beam and a spherical thin shell might be permitted. An advantage of relativistic beaming is a smaller required luminosity at the source because the observed flux, $\phi \sim \Gamma^{2} L /$ $4 \pi d^{2}$, is enhanced by a solid angle factor, $\Gamma^{2}$ (Krolik \& Pier 1991). However, the number of sources must be a factor $\Gamma^{2}$ higher in order to account for the observed number of GRBs. In the case of cosmological GRBs, this factor could be as high as $10^{6}$ for the above limits on $\Gamma$, which is unacceptably large for many models. Source geometries with beaming angles larger than $1 / \Gamma$ might therefore be preferred.

\subsection{Source Models}

The results on the isotropy and count spectrum of GRBs reported by BATSE has caused a dramatic shift in thinking about burst origins and in theoretical modeling. The literature has exploded with new ideas on how to make a GRB with much larger energy than previously believed, as well as reexamination of many old ideas. The current debate focuses on whether GRB sources are in an extended Galactic halo or are at cosmological distances. I will discuss a sampling of the published models, the unsolved problems, and the predictions for observations that may distinguish between the two types of model.

\subsubsection{Galactic Models}

Although extended Galactic halo distributions have been shown to be consistent with the presently observed level of 
isotropy, these models must live with the possibility that BATSE will continue to improve their isotropy measurements and require even larger halos. These models are attractive because they can preserve some features of the Galactic neutron star models studied over the last two decades. In fact it is implicitly assumed in the papers which calculate expected distributions of high-velocity neutron stars in the Galactic halo and show consistency with the BATSE data (Li \& Dermer 1992; Eichler \& Silk 1992) that one or more of the GRB mechanisms proposed for neutron stars in the disk will work for those in the halo. However, this assumption may be overly optimistic for several reasons. One is that the higher luminosity, $L \sim 10^{42}$ $10^{43} \mathrm{ergs} \mathrm{s}^{-1}$, of GRBs at a distance $\sim 100 \mathrm{kpc}$ is about $10^{4}$ times the Eddington luminosity for a neutron star, unless the radiation is highly beamed. Static source models will therefore not apply, since radiation pressure would drive a wind away from the star. For example, the cyclotron line models which so successfully accounted for the observed Ginga features (Wang et al. 1989) assumed static slab scattering regions. It has not been fully demonstrated that these features can be produced in a wind driven by radiation pressure although some attempts have been made in this direction (Miller et al. 1991). Furthermore, since $\tau_{\gamma \gamma} \gg 1$ in halo sources with isotropic radiation, fireballs will form unless the radiation is beamed or there is relativistic motion of the source.

Halo models predict that an anisotropy (a dipole moment in the direction of the Galactic center) will eventually be detected in the observed burst distribution. The anisotropy should appear first in the distribution of the brightest bursts, since they are the nearest sources, whose distances are comparable to our distance from the Galactic center. It may require several more years of BATSE observations to detect enough of these bright sources. However, a distribution of 60 bright GRBs published by Atteia et al. ( 1987) from many years of observation showed no anisotropy. In contrast, for a disk population the anisotropy is expected to appear first in the faintest bursts which have distances larger than the disk scale height.

\subsubsection{Cosmological Models}

Cosmological source models share some of the same problems of halo models, such as high pair production optical depth and even higher luminosities, and encounter a few additional difficulties. One of these is the very short burst fluctuation time scales, which in a static source implies a size typical of neutron stars. Models involving larger sources, such as the massive black holes in active galactic nuclei, must have extreme relativistic motion of the source to produce the required time scales. Another problem is that nearly all of the models assume sources are in galaxies, and no galaxies have been observed down to 23d magnitude in GRB error boxes (Schaefer 1991).

One popular and intriguing model proposed for cosmological GRBs is the merging of two neutron stars or of a neutron star and a black hole (Paczyński 1986, 1991b; Eichler et al. 1989; Mészáros \& Rees 1992). The rate of neutron star mergers is estimated to be $\sim 10^{-6} \mathrm{yr}^{-1}$ galaxy ${ }^{-1}$ from the number of binary neutron star systems (four) observed in our own Galaxy, their implied birthrate, and the projected time scale for gravitational radiation decay of the orbit (Narayan, Piran, $\&$ Shemi 1991). There should then be $\sim 100$ mergers per year out to a distance of $1 \mathrm{Gpc}$ and $10^{3}-10^{4}$ per year out to the horizon, easily enough to account for the observed rate of GRBs. The binding energy release in a binary neutron star merger is $\sim 10^{53}-10^{54}$ ergs, most of which will appear as gravitational waves $\left(8 \times 10^{52}\right.$ ergs $)$ and neutrinos $\left(2 \times 10^{53} \mathrm{ergs}\right)$ on a time scale of a few milliseconds (Clark \& Eardley 1977). A mechanism for producing GRBs from neutron star mergers was suggested by Eichler et al. (1989), who proposed that neutrinos and antineutrinos would annihilate in the reaction, $\nu+$ $\bar{\nu} \rightarrow e^{+}+e^{-} \rightarrow \gamma \gamma$, to produce $\gamma$-rays. The efficiency of this process is about $10^{-3}$, so that $\sim 10^{50}-10^{51}$ ergs should appear in $\gamma$-rays.

The release of $10^{51}$ ergs in $\gamma$-rays in a neutron star merger will produce a relativistic fireball with at least some baryon contamination (Levinson \& Eichler 1993): The problem is then how do neutron star mergers make GRBs when almost all of the energy in the fireball goes into accelerating the baryons. Another problem with the neutron star merger models is that the time scale of a few milliseconds for collapse to a black hole is much shorter than observed GRB time durations. A solution to both, suggested by Mészáros \& Rees (1993), is that the fireball convert its kinetic energy into a GRB through interaction with an external medium. This would involve formation of both a shock ahead of the contact discontinuity as the fireball slows down by sweeping up material and a reverse shock moving back into the fireball plasma. The reverse shock may produce a nonthermal radiation spectrum through diffusive shock acceleration of particles. The fireball has expanded to a radius of $r_{d} \sim 10^{16}-10^{17} \mathrm{~cm}$ at this point, and the observed burst duration will be $\sim r_{d} c^{-1} \Gamma^{-2} \sim 1$ s for $\Gamma \sim 10^{2}-10^{3}$ because the fireball appears to be compressed into a thin shell.

Other cosmological models for GRBs involve active galactic nuclei as the energy source. Prilutsky \& Usov (1975) proposed some time ago that the collapse of supermassive stars at the centers of galaxies could provide enough energy for a GRB at cosmological distances. Alternatively, GRBs could be phenomena associated with jets in active galaxies, with beamed $\gamma$-rays produced either through synchrotron (Brainerd 1992b) or synchrotron self-Compton (McBreen, Plunkett, \& Metcalfe 1993) radiation in the jet.

Woosley (1993) considers the formation of a transient accretion disk around a black hole, which would enable the energy to be released on a viscous time scale of a few seconds. The black hole could result from a neutron star or neutron starblack hole merger but more often will result from a failed Type lb supernova, one where the envelope does not explode but collapses back onto the core. These events will release much more energy than neutron star mergers and could produce bursts with luminosity $\sim 10^{50}$ ergs.

It has also been suggested by Usov (1992) that cosmological GRBs could be produced by the rapid spin down of a millisecond pulsar with a very high $\left(10^{15} \mathrm{G}\right)$ magnetic field. Such pulsars would be formed by the collapse of accreting white dwarfs with anomalously high magnetic fields of $\sim 10^{9} \mathrm{G}$. Both the rate of magnetic dipole spin-down energy loss of

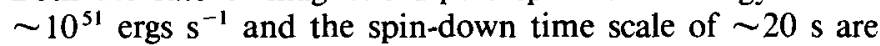
consistent with a GRB. A relativistic fireball will also result in this model.

There are a number of observational effects predicted by cosmological source models. A source at a cosmological dis- 
tance has a $0.5 \%$ probability of being gravitationally lensed by an intervening galaxy (Turner, Ostriker, \& Gott 1984). Thus BATSE should detect a lensed GRB in a few years of observation. The resulting multiple images of a lensed burst would not be detectable, but the occurrence of two bursts from the same location in the sky, identical in both spectrum and time history and separated by about a month, would be strong evidence of gravitational lensing (Paczyński 1986; Mao 1992). If GRBs are in or are associated with galaxies then the sources should be clustered like galaxies, that is, they should have the same twopoint angular correlation. To test this prediction will require several more years of data accumulation by BATSE. The source spectra should also exhibit redshift effects such as a hardness/intensity correlation, but so far the evidence for such a correlation is weak and probably inconclusive (Pacsiesas et al. 1992). If GRBs are the result of neutron star mergers then the associated gravitational waves should be seen with future detectors such as LIGO. If GRB detectors that are sensitive down to $0.1 \mathrm{keV}$ can be designed and flown they should detect photoelectric absorption in GRB spectra below 1-2 keV as a function of Galactic latitude (Paczyński 1991b; Schaefer 1993). Finally, galaxy counterparts should eventually be detected in GRB error boxes. Assuming a GRB luminosity of $6 \times$ $10^{50}$ ergs s $^{-1}$, derived from cosmological model fits to their combined BATSE-PVO count spectrum, and Schaefer's (1991) apparent magnitude limits Fenimore et al. (1993b) conclude that host galaxies must have absolute visual magnitude fainter than -18 . This would rule out active galactic nuclei and Seyfert galaxies but would allow faint, normal galaxies.

\subsection{One Population or Two?}

Even though both extended Galactic halo and cosmological source distributions for GRBs can account for the spatial distribution and count spectrum observed by BATSE, it is not clear whether either can account for the previously existing evidence for nearby Galactic neutron stars discussed in $\S 2$, most notably cyclotron lines. The need to reconcile two sets of seemingly incompatible observed characteristics of GRBs has led to consideration of two-population models. Higdon \& Lingenfelter (1992) propose two Galactic populations of neutron stars, one in the disk with average luminosity $L_{1}$ and maximum distance $\left(d_{\max }\right)_{1}$ less than the disk scale height and one in the halo with $L_{2} \gg L_{1}$ and $\left(d_{\max }\right)_{2} \gg\left(d_{\max }\right)_{1}$. Smith \& Lamb (1993) show that a combined disk plus halo model for GRB sources can fit the BATSE count spectrum, with as many as $2 / 3$ of the observed bursts in the disk depending on the type of halo distribution.

Several problems with two population models have been pointed out. One is that there is no strong evidence of two populations with very different luminosities and distances in either the observed spectral or temporal behavior of GRBs. Another problem, noted by Paczyński (1992), is that the count spectrum of two population models such as the one proposed by Higdon \& Lingenfelter (1992) will always be dominated at low fluence by the nearby population having a steep count spectrum and at high fluence by the distant population having a flat count spectrum. The composite count spectrum is inconsistent with the count spectrum observed by BATSE which is flat at low fluence and steep at high fluence.
These problems, however, are not necessarily fatal. As is illustrated in Figure 1, if the minimum fluence to which BATSE is sensitive falls near but above the fluence at which the nearby population begins to dominate the count spectrum, then the composite count spectrum could be consistent with that observed. There could even be nearly equal numbers of nearby and distant sources observed, even though the entire count spectrum is dominated by the distant sources. This is, in fact, the case in Smith \& Lamb's (1993) models. There is some suggestion that the distribution of GRB durations is bimodal both in BATSE data (Kouveliotou et al. 1993) and in data from earlier detectors (Hurley 1992), with the two groups having durations $\sim 0.1-1 \mathrm{~s}$ and $\sim 1-100 \mathrm{~s}$. Whether these groups have different fluence or spectral characteristics is not yet clear, although there may be hints in this direction (Lamb \& Graziani 1993).

\section{CONCLUSION}

The present status of the theory of GRBs is one of considerable puzzlement but also one of excitement. With the sources of the bursts completely unknown, their distances uncertain by six orders of magnitude and their energies uncertain by 12 orders of magnitude, models are in a fairly primitive state. The question of which mechanisms are operating to accelerate particles in GRB sources thus seems far from being answered. Furthermore, GRB spectra continue to be observed up to energies of $100 \mathrm{MeV}$ and higher (Schneid et al. 1992; Sommer et al. 1993) so we have not even seen the limit to the required particle energies. The pressing issues over the next few years will be making predictions and observations that will constrain the distances to GRBs and at least answer the question of whether or not they are cosmological. Of course the question may not be quite so simple, as I believe that a two (or more) population model exists as a real possibility. It will become more convincing if BATSE or one of several other GRB detectors to be launched in the near future confirms the cyclotron lines seen by Ginga.

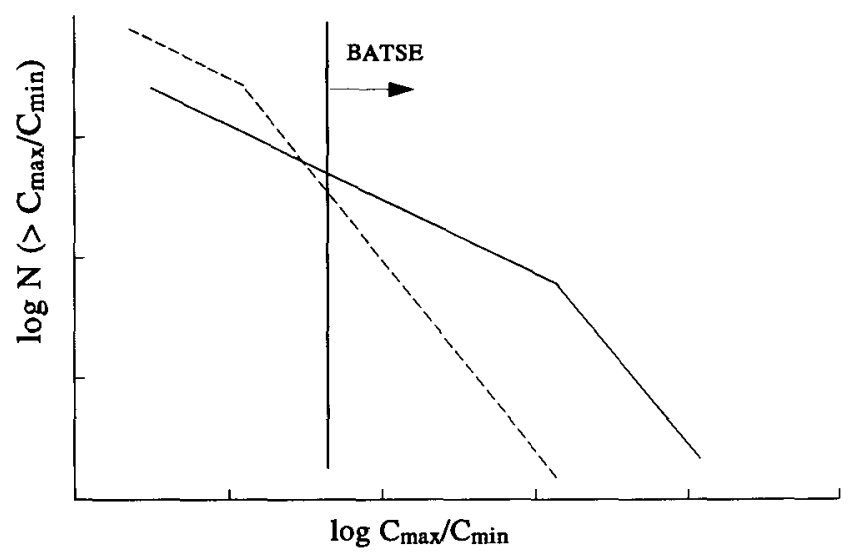

FIG. 1.-Hypothetical count spectra for two populations of GRBs. The dashed line could represent a relatively nearby population of sources having low luminosities, and the solid line could represent a more distant population having much higher luminosities. If the sensitivity limit of the BATSE detector (vertical line) falls above the $C_{\max } / C_{\min }$ value where two count spectra cross, then the combined count spectrum could be consistent with that observed. 
Atteia, J. L., et al. 1987, ApJS, 64, 305

Baring, M. G. 1993, ApJ, in press

Baring, M. G., \& Harding, A. K. 1993, in Proc. 23d Internat. Cosmic Ray Conf. (Calgary), in press

Brainerd, J. J. 1992a, Nature, 355, 522 - 1992b, ApJ, 394, L33

Clark, J. P. A., \& Eardley, D. M. 1977, ApJ, 215, 311

Dermer, C. D. 1992, Phys. Rev. Lett, 68, 1799

Eichler, D., Livio, M., Piran, T., \& Schramm, D. N. 1989, Nature, 340, 126

Eichler, D., \& Silk, J. 1992, Science, 257, 937

Fenimore, E. E., et al. 1992, Nature, 357, 140

Fenimore, E. E., Epstein, R. I., \& Ho, C. 1993a, A\&AS, 97, 59

Fenimore, E. E., et al. 1993b, Nature, submitted

Goodman, J. 1986, ApJ, 308, L47

Hakkila, J., et al. 1993, in Proc. Compton Symposium, ed. N. Gehrels (New York: AIP), in press

Harding, A. K. 1991, Phys. Rep., 206, 327

Harding, A. K., \& Preece, R. D. 1989, ApJ, 338, L21

Harrison, P. A., Lyne, A. G., \& Anderson, B. 1993, MNRAS, in press

Hartmann, D. H., Linder, E. V., \& The, L. 1993, in Proc. Compton Symposium, ed. N. Gehrels (New York: AIP), in press

Heuter, G. J. 1988, in Nuclear Spectroscopy of Astrophysical Plasmas, ed. N. Gehrels \& G. H. Share (New York: AIP), 285

Higdon, J. C., \& Lingenfelter, R. E. 1992, Nature, 356, 132

Hurley, K. 1992, in Gamma-Ray Bursts, ed. W. S. Paciesas \& G. J. Fishman (New York: AIP), 3

Kouveliotou, C., et al. 1993, in Proc. Integral Workshop, in press

Krolik, J. H., \& Pier, E. A. 1991, ApJ, 373, 277

Kwok, P. W., et al. 1993, in Proc. Compton Symposium, ed. N. Gehrels (New York: AlP), in press

Lamb, D. Q., \& Graziani, C. 1993, ApJ, submitted

Levinson, A., \& Eichler, D. 1993, ApJ, in press

Li, H., \& Dermer, C. D. 1992, Nature, 359, 514

Lyne, A. G., Anderson, B., \& Salter, M. J. 1982, MNRAS, 201, 503

Mao, S. 1992, ApJ, 389, L41

Mao, S., \& Paczyński, B. 1992, ApJ, 388, L45

\section{REFERENCES}

Mazets, E. P., et al. 1981, Nature, 290, 378

McBreen, B., Plunkett, S., \& Metcalfe, L. 1993, in Proc. Recent Advances in High Energy Astronomy (Toulouse), in press

Meegan, C. A., et al. 1991, Nature, 335, 143 1993, IAU Circ., No. 5641

Mészáros, P., \& Rees, M. J. 1992, ApJ, 397, 570 1993, ApJ, 405, 278

Miller, G., et al. 1991, Phys. Rev. Lett, 66, 1395

Murakami, T., et al. 1988, Nature, 335, 234

- 1991, in Gamma-Ray Bursts: Observations, Analyses and Theories, ed. C. Ho, R. I. Epstein, \& E. E. Fenimore (Cambridge: Cambridge Univ. Press), 239

Narayan, R., Piran, T., \& Shemi, A. 1991, ApJ, 379, L17

Paciesas, W. S., et al. 1992, in Gamma-Ray Bursts, ed. W. S. Paciesas \& G. J. Fishman (New York: AIP), 190

Paczyński, B. 1986, ApJ, 308, L43

- 1991a, A\&A, 41, 157

- 1991 b, A\&A, 41, 257 1992, A\&A, 42, 1

Piran, T. 1992, ApJ, 389, L45

Piran, T., \& Shemi, A. 1992, ApJ, 403, L67

Prilutsky, O. F., \& Usov, V. V. 1975, Ap\&SS, 34, 395

Schaefer, B. E. 1991, in Gamma-Ray Bursts: Observations, Analyses and Theories, ed. C. Ho, R. I. Epstein, \& E. E. Fenimore (Cambridge: Cambridge Univ. Press), 107

_ 1993, in Proc. Compton Symposium, ed. N. Gehrels (New York: AIP), in press

Schaefer, B. E., et al. 1992, ApJ, 393, L51

Schmidt, W. K. H. 1978, Nature, 271, 525

Schneid, E., et al. 1992, A\&A, 255, L13

Shemi, A., \& Piran, T. 1990, ApJ, 365, L55

Smith, I. A., \& Lamb, D. Q. 1993, ApJ, 410, L23

Sommer, M., et al. 1993, ApJ, submitted

Turner, E. L., Ostriker, J. P., \& Gott, J. R. 1984, ApJ, 283,

Usov, V. V. 1992, Nature, 357, 472

Wang, J. C. L., et al. 1989, Phys. Rev. Lett, 63, 1550

Woosley, S. E. 1993, ApJ, 405, 273 